\title{
Stato e mercato: una storia contemporanea
}

\author{
di Emanuele Felice
}

Feb 12, 2021 | Emergenza e (nuova) normalità, In evidenza, Stato/Mercato $\mid \underline{0 \mid}$

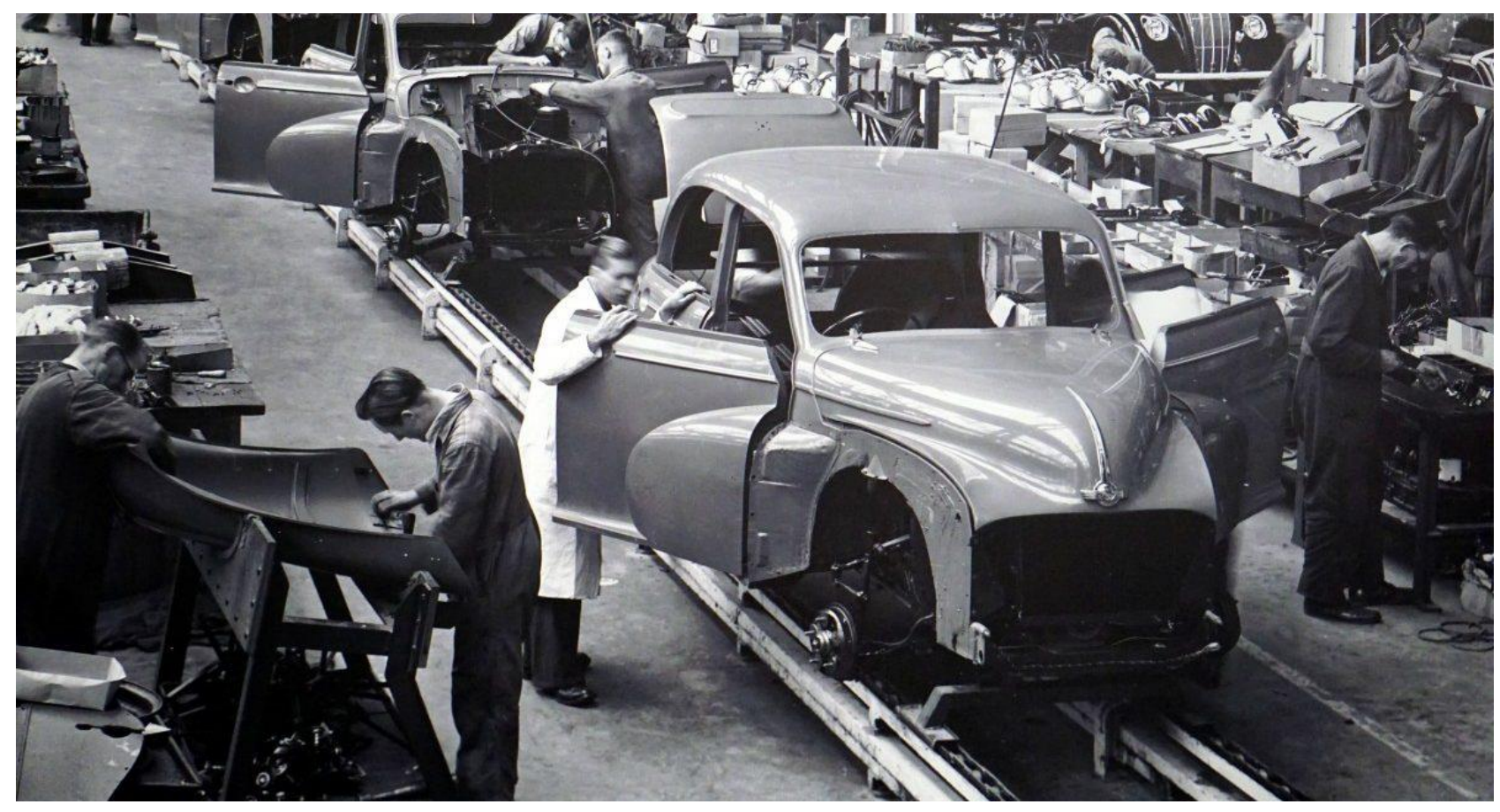

Il dossier sulla Summer school 2020

si compone di 5 coppie di parole chiave.

Questo testo afferisce alla coppia di parole chiave

Stato/Mercato

\begin{abstract}
Nella quarta giornata della Summer School 2020 abbiamo chiesto a Emanuele Felice e Lea Ypi di riflettere con noi sulle parole chiave Stato e Mercato.

Emanuele Felice ci invita a ripercorrere la storia dei rapporti fra Stato e mercato in Europa a partire dalla Seconda rivoluzione industriale, quando la visione liberale classica si modifica a causa della scelta protezionista adottata dagli Stati per lo sviluppo dell'industria nazionale. Durante la Grande Guerra, l'interventismo dello Stato inevitabilmente si accentua, in ragione della necessità di centralizzare e organizzare le risorse in vista dello sforzo bellico. In seguito, con la fase keynesiana, che ha origine negli '30 e si dispiega dopo la Seconda guerra mondiale, lo Stato assume i ruoli di produttore, programmatore e redistributore divenendo un attore economico di primo piano. Si tratta di un modello di successo (si pensi al welfare state) che viene però scardinato nella fase successiva quando, a partire dagli anni '70, si assiste a un ridimensionamento del ruolo dello Stato. Nell'ultimo decennio, però, tale cornice neoliberista è entrata in crisi a causa della crisi economica, democratica, ambientale e ora anche sanitaria.
\end{abstract}

L’intervento pubblico in economia: i primi passi 
La questione del ruolo che può e dovrebbe svolgere lo Stato nell'economia è tornata alla ribalta nell'ultimo decennio, sia a causa delle conseguenze della crisi globale del 2008 sia, più di recente, a causa dell'emergenza pandemica, che ha posto in modo drammatico il tema della mobilitazione di enormi risorse pubbliche per evitare il collasso sanitario ed economico.

Per capire meglio in che modo e in che cosa dovrebbe realizzarsi l'intervento pubblico, oggi invocato anche da molti fautori del liberalismo, può essere utile ripercorrere la storia del rapporto fra Stato e mercato in Occidente a partire dalla Seconda rivoluzione industriale.

Nella prima fase dello sviluppo economico moderno, la prospettiva di politica economica che prevale è quella dello "Stato minimo" (in particolare nell'Ottocento): lo Stato deve garantire le condizioni fondamentali per le attività della borghesia in espansione, ovvero la legge, lo stato di diritto, la banca centrale, una moneta affidabile, eventualmente le infrastrutture.

Questa visione, propria del liberalismo classico, si trova fin da subito temperata da una questione pratica. Lo sviluppo economico moderno, infatti, nasce e decolla sulle ali delle borghesie, che si identificano con gli stati nazionali. I quali, non a caso, nascono a loro volta, e prendono forma proprio alla vigilia del «decollo industriale» (ne sono esempi il Regno d'Italia, l'Impero tedesco, la Monarchia duale austro-ungarica, la Repubblica francese, gli Stati Uniti dopo la guerra civile, il Giappone con la rivoluzione Meiji). Tutti questi Paesi si trovano di fronte al problema di come avviare un processo di sviluppo che, sebbene la teoria di matrice anglosassone non lo preveda, di fatto non può realizzarsi, fin dall'inizio, se non attraverso un certo intervento dello Stato. Intervento protezionista: ovvero l'imposizione di tariffe per limitare l'importazione di prodotti inglesi, che costano meno perché costano meno (fabbricati con le macchine), e per favorire lo sviluppo di un'industria domestica. Anche se si tratta di un dato storico che spesso tendiamo a dimenticare, il protezionismo è la strategia con cui i grandi paesi inseguitori dell'Inghilterra si sono industrializzati. Insomma, l'idea che la politica dovesse orientare e favorire lo sviluppo industriale c'è stata fin dal principio.

Peraltro, in riferimento a quel periodo, ancora non si può parlare di un intervento dello Stato nell'economia come l'avremmo conosciuto in seguito. Tale possibilità si fa strada a poco a poco nel pensiero liberale progressista (il cosiddetto "nuovo liberalismo" inglese, per esempio), con le tesi di coloro (per l'Italia, pensiamo a Giolitti e ancor più a Nitti) secondo cui lo Stato dovrebbe intervenire attivamente nell'economia per rimuovere quegli ostacoli che impediscono l'accesso di tutti all'attività economica, la piena realizzazione del potenziale economico dei singoli e quindi della collettività (che è la somma della realizzazione degli individui). Si comincia a parlare di intervento dello Stato nei servizi essenziali - quelli destinati a promuovere, usando un linguaggio tipicamente progressista, l'emancipazione umana (l'istruzione, la sanità) - ma anche in altri tipi di imprese economiche. Ad esempio, in epoca giolittiana si nazionalizzano le ferrovie (1905), cosa che peraltro determina una nuova fase di miglioramento della rete ferroviaria; poi, con Nitti, arriveranno le prime leggi per l'industrializzazione e lo sviluppo del Mezzogiorno.

Abbiamo fin qui raccontato il modo in cui l'intervento pubblico inizia a farsi strada nella strategia di sviluppo economico delle singole nazioni europee. Peraltro, la dimensione statuale risulta decisiva anche per la proiezione di potenza delle borghesie nazionali, che di questo sviluppo sono in un certo senso l'incarnazione. 
Con la Grande guerra, il rapporto fra Stato ed economia fa un salto di qualità. Durante il primo conflitto mondiale, infatti, lo Stato, in tutti i Paesi, entra come attore di primissimo piano per organizzare lo sforzo bellico, che richiede un coordinamento centrale (si pensi alle commesse di guerra). Anche l'arrivo dell'occupazione femminile nell'industria, per il «fronte interno», è un processo gestito dallo Stato.

Il ruolo del Pubblico si accentua, poi, nell'immediato dopoguerra, quando lo Stato, in molti Paesi, interviene per salvare le imprese in difficoltà con la riconversione. Entriamo così in una seconda fase, che dura dal periodo infrabellico fino agli anni Settanta, nella quale è ampiamente presente l'intervento diretto dello Stato per lo sviluppo dell'economia, a fianco ma a volte anche in aperta sostituzione dei privati.

Ne è il presupposto, sul piano della teoria, il cosiddetto keynesismo, che prende piede negli Stati Uniti e in Gran Bretagna dopo la crisi del 1929. Anche questa fase si dispiega saldamente nella cornice nello Stato nazione, il soggetto che si fa carico dell' intervento pubblico.

Negli anni Trenta, peraltro, gli stati liberali sono alle prese con due temibili sfidanti, anche sul piano economico, l'Urss di Stalin e il modello nazista e fascista (che pure prevede un maggiore intervento dello Stato).

Dopo la Seconda guerra mondiale, quando l'alternativa nazifascista, sconfitta militarmente, viene a cadere - e rimane soltanto la competizione con il mondo comunista dell'Urss e dei suoi stati satelliti - in tutto l'Occidente prevale un modello di economia mista in cui lo Stato assume altri ruoli, oltre a quelli consueti di garanzia e di sicurezza: il ruolo di produttore economico (anzitutto nei settori strategici ma a volte anche in una congerie di altre attività, a seconda dei paesi); il ruolo di programmatore economico (prendendo esempio dall' esperienza dei piani quinquennali sovietici, riadattati per le economie di mercato); il ruolo di redistributore. Uno Stato, insomma, che oltre a garantire le condizioni essenziali per gli operatori privati, diviene esso stesso un attore economico di primo piano; in più, programma lo sviluppo economico e redistribuisce la ricchezza, anche attraverso la fornitura di servizi essenziali come la sanità, la sicurezza sociale, l'istruzione (welfare state).

Questo modello di successo, che nel secondo Novecento ha creato in Occidente le società di maggiore benessere (in termini di diritti e di opportunità) che mai si siano viste in tutta la storia umana, viene però in parte scardinato, dagli anni Settanta in avanti.

Si ridimensiona, cioè, l'idea che lo Stato debba programmare, produrre e redistribuire, mentre rimane l'idea che lo Stato debba garantire le condizioni essenziali per l'attività privata. Dagli anni Settanta, insomma, c'è dunque un ritorno al modello, per così dire, liberale classico.

Le ragioni sono complesse, in parte di natura tecnologica (come la diffusione dei sistemi di automazione che, riducendo il peso del lavoro umano nella produzione, indeboliscono anche la forza contrattuale dei lavoratori), in parte di natura politica (difficoltà a governare il processo di globalizzazione, nel quale lo Stato nazione si trova ad avere un potere sempre più ridotto). Naturalmente anche la teoria economica - detta appunto neo-liberale - non manca di mettere in discussione l'idea di Stato programmatore, produttore e a redistributore. Questo passaggio avviene soprattutto in USA e nel Regno Unito, va detto, meno nell'Europa continentale.

Negli ultimi anni, però, la cornice neo-liberale ha cominciato a entrare in crisi, prima ancora dello scoppio dell'attuale pandemia (anche se poi il Covid ha accentuato questo processo): perché, tolta allo Stato l'attività di programmazione, il capitalismo di mercato, da solo, non pare essere in grado 
di affrontare il problema della transizione ecologica (cioè di evitare la catastrofe ambientale). Inoltre, si è dovuto prendere atto che il mercato da solo non riesce a ridurre le diseguaglianze fra le persone, che, in assenza di un efficace Stato redistributore, tendono invece ad aumentare, impedendo anche la crescita economica.

C'è poi un terzo motivo di crisi dell'ideologia neo-liberale, forse meno evidente ma altrettanto importante: il ritrarsi della politica dall'economia porta a un'assolutizzazione della sfera economica rispetto alla dimensione politico-democratica e questo, nei Paesi che non hanno conosciuto la democrazia liberale, comporta inevitabilmente una crescita economica senza diritti umani. Tutto ciò mentre il paradigma neo-liberale sosteneva che la crescita economica portasse quasi automaticamente con sé un miglioramento nei diritti dell'uomo. Adesso stiamo scoprendo che non è così.

\section{L' Italia, un caso paradigmatico}

Il caso Italia rientra pienamente nella storia di cui abbiamo appena parlato.

In particolare, è paradigmatico quello che si verifica dopo la Seconda guerra mondiale, cioè il "miracolo economico": venticinque anni, fra il 1948 e il 1973, in cui il nostro è uno dei Paesi del mondo avanzato (insieme alla Germania e al Giappone) che cresce di più.

Il miracolo economico si fonda sull'intervento dello Stato e sulla collaborazione fra privato e pubblico, particolarmente efficaci, l'uno e l'altra, negli anni Cinquanta (già un po' meno nei Sessanta). Ne sono inizialmente protagoniste grandi imprese pubbliche come l'IRI (in diversi settori di base, dalla cantieristica alla siderurgia) e l'ENI (nell'energia). Quindi l'ENEL, che nasce nel 1962 per nazionalizzare l'energia elettrica e portarla così ovunque, anche nei piccoli paesi montani, supplendo ai fallimenti del mercato.

Lo Stato produttore e lo Stato programmatore, poi, si mescolano, come nel caso della Cassa per il Mezzogiorno, dove le imprese private chiedono al Pubblico di programmare, di realizzare infrastrutture di base e di fornire incentivi, i quali naturalmente vengono poi dati anche alle imprese pubbliche, che devono per legge riservare al Sud il 60\% dei nuovi investimenti. Quello che invece ha funzionato meno bene, in Italia, è stato il ruolo di Stato redistributore: il nostro Paese ha sempre avuto un welfare imperfetto, frammentato e in parte "clientelare".

Anche in Italia la creazione del welfare risale, prima ancora che al fascismo, come comunemente si crede, all'inizio del Novecento, alla sinistra liberale (ai governi Giolitti e Nitti). Si trattava, allora, soltanto di un embrione, che sarà potenziato soprattutto negli anni Sessanta e Settanta. Tuttavia, il nostro Paese non ha mai avuto un sistema di welfare universalistico e coerente come quello dei paesi del Nord e Centro Europa. E bisogna dire che, mentre le classi dirigenti sono state tutto sommato abbastanza capaci nell'esercitare il ruolo di Stato produttore (almeno durante il miracolo economico), in quello di Stato programmatore non lo sono state altrettanto (la nostra programmazione negli anni Sessanta è stata un relativo insuccesso, a differenza di quella francese). Quando il rapporto fra le imprese pubbliche e la politica, organizzata in partiti di massa capaci di fornire una buona classe dirigente, ha nel complesso funzionato; quando la politica non ha imposto le sue decisioni alle imprese pubbliche, questa economia mista è stata utile per lo sviluppo del Paese e decisiva nei settori chiave (dall' acciaio alle infrastrutture, alla stessa modernizzazione del Sud Italia). 
Successivamente, e in parallelo con il declino dei partiti (specie di maggioranza), divenuto patologico negli anni Ottanta (per il clientelismo e per la corruzione, per non parlare delle infiltrazioni della grande criminalità), l'Italia ha conosciuto una degenerazione del ruolo dello Stato più forte che altrove, accompagnata da una crescita esplosiva del debito pubblico, che è diventato un fardello per lo sviluppo economico. Da qui una reazione antistatalista, forse più accentuata che in altri paesi.

In questo recente discredito, spesso ideologico, in cui cade l'idea di Stato, non bisogna però trascurare anche una motivazione politica.

Il PCI, il maggiore partito di opposizione nella Prima repubblica, poi PDS e DS nella Seconda, dopo il fallimento del comunismo si converte, a tratti con entusiasmi da neofita, alle (dominanti) idee neo-liberali. Trascura, invece di coltivare, un forte pensiero socialista e riformatore, forse mai pienamente sviluppato neanche in passato (quando appunto era stato un partito ideologicamente comunista). Se invece già nella Prima repubblica vi fosse stata una vera alternativa socialista, non obbligata a farsi perdonare il fallimento del comunismo, forse vi sarebbe stato anche un pensiero più critico nei confronti del neo-liberalismo. Peraltro, va detto che anche in Paesi dove il pensiero socialdemocratico era presente con forza gli storici partiti socialisti hanno ceduto alla fascinazione neo-liberale. Resta il fatto che negli anni Novanta in Italia la classe dirigente dell'ex PCI non è stata capace di elaborare una visione alternativa a quella neo-liberale ed ha sottovalutato l'importanza del contributo che il pensiero socialista italiano avrebbe potuto, a questo scopo, fornire.

L'Italia negli anni Novanta e Duemila viene così a caratterizzarsi per un'egemonia culturale antistatalista, che comporta anche da noi un significativo ridimensionamento del ruolo dello Stato; non certo come negli Usa e in Gran Bretagna, ma più propriamente in linea con gli altri paesi dell'Europa continentale (grossomodo, a un livello intermedio tra la Francia e la Germania).

Per l'Italia, una delle conseguenze del ridimensionamento del ruolo dello Stato, accompagnato dalle politiche di austerity per rientrare dal debito pubblico, è stata la perdita di competenze della pubblica amministrazione, specie nell'attività di programmazione (ma non solo); con conseguenze negative anche per le imprese private, che ancora oggi si trovano a mancare di un ausilio fondamentale.

\section{Stato, mercato e pandemia}

La pandemia ha accentuato la crisi della visione neo-liberale, mostrando come il ruolo del Pubblico sia davvero indispensabile.

Prima di tutto, l'emergenza sanitaria ha reso evidente come ci possano essere anche cose più importanti dell'economia: la vita e la salute dei cittadini. E questo vuol dire che la sanità pubblica torna a essere un valore in sé, non subordinabile alle logiche del profitto. Dopo aver privatizzato, «razionalizzato» in molti ambiti, si è tornati in tutta Europa a rivalutare la sanità pubblica e i diritti sociali che ne sono il fine e che vanno garantiti a prescindere. Si è poi palesata l'importanza dello Stato nel sostenere alcuni settori in crisi e nel garantire la tenuta sociale.

L'Italia è entrata in questa pandemia con un sistema di welfare frammentato (retaggio del passato, come si è visto) e, nel corso del 2020, ha fatto alcuni importanti passi in direzione di un welfare universalistico (dando per esempio la cassa integrazione anche alle imprese che hanno cinque o meno dipendenti, o predisponendo le prime forme di tutela per le partite Iva). 
Si tratta di una tendenza comune a tutti i paesi economicamente più avanzati. La stessa Unione Europea ha risposto alla crisi avviando un significativo ripensamento del suo modello, che non a caso, negli anni Novanta, era stato costruito dentro la visione, allora egemone, di uno Stato minimo (l'Unione doveva cioè garantire le condizioni essenziali per la libertà d'impresa e di mercato, e poco altro). Per esempio, l'Europa non poteva fare debito (gli eurobond, fino alla crisi del Covid, erano impensabili), non poteva promuovere singoli settori industriali o aiutare particolari imprese considerate strategiche. Di più: impediva che anche i singoli stati intervenissero nell'economia (la normativa contro gli aiuti di Stato) o facessero debito. Ora tutto questo è sospeso, almeno temporaneamente.

Con il Next generation EU vediamo l'Europa svolgere un ruolo attivo (che vuol dire fare debito) nel promuovere la transizione energetica e l'innovazione tecnologica, e anche nel combattere le disuguaglianze. L'Europa, pare, sembra interessata a utilizzare questa crisi per elaborare un nuovo modello di sviluppo in cui l'intervento pubblico (che non è più solo dello Stato nazionale) possa servire per governare lo sviluppo tecnologico in direzione dei diritti umani, complessivamente intesi (civili, sociali e ambientali). Ma è una sfida aperta, tutt'altro che scontata.

In Italia possiamo trarre grandi benefici da questo cambio di paradigma, a condizione però di attrezzarci al meglio per cogliere le nuove opportunità. Per farlo, dobbiamo:

1. Restituire alla pubblica amministrazione la capacità di programmare. Con il Next generation $E U$ è tornato lo Stato programmatore, anche se non lo Stato imprenditore. Ma sorge una domanda: la nostra amministrazione pubblica, da quasi trent'anni concentratasi sui tagli, è in grado di programmare la spesa?

2. Sintonizzarsi anche culturalmente con questi tempi nuovi, con quello che sta accadendo. In Italia, la reazione antistatalista è stata più forte che altrove, forse per una debolezza culturale che ha riguardato, come si è visto, anche la sinistra riformista. Guardando all'attuale dibattito pubblico, specie fra gli opinionisti, e confrontandolo con le dichiarazioni di Ursula Von der Leyen (non certo un'esponente della sinistra radicale), l'impressione è che buona parte dell'intellettualità italiana sia rimasta indietro, per così dire, rispetto alle sfide poste dalla nuova situazione. Si pensi al tema della transizione energetica; oppure dell'istruzione: fra i paesi avanzati, siamo quelli che investono meno in rapporto al PIL e questo è molto preoccupante. Più in generale, se è vero che negli ultimi decenni abbiamo perso il treno dell'automazione e della telematica, possiamo però avviare ora una nuova stagione di crescita economica (e tecnologica) grazie alla transizione energetica, alla biogenetica, alle scienze mediche applicate alla cura della persona. Occorre però mettere a sistema e valorizzare le nostre imprese pubbliche, che sono nelle condizioni di svolgere a questo scopo un ruolo decisivo importante.

3. Avere la consapevolezza, infine, che sul nostro declino pesano moltissimo tre tipi di diseguaglianze, che caratterizzano l'Italia più di altri paesi dell'eurozona e si aggiungono, attraversandoli, ai divari di reddito: le disuguaglianze di generazione, a danno dei giovani che infatti emigrano; di genere, a danno delle donne; geografiche, soprattutto fra Nord e Sud.

La nuova fase potrebbe portare al nostro Paese addirittura più benefici che altrove in Europa; d'altra parte (e proprio per questo), c'è il rischio che possa tramutarsi in una grande occasione persa. Per il momento, registro che ci si 